\title{
WEAKLY QUASISYMMETRIC EMBEDDINGS OF R INTO C
}

\author{
M. JEAN MCKEMIE and JEFFREY D. VAALER*
}

\section{Introduction}

In this note we classify all continuous functions $f: \mathbf{R} \rightarrow \mathbf{C}$ which satisfy

$$
|f(x+y)-f(x)|=|f(x-y)-f(x)|
$$

for all $x$ and $y$ in $\mathbf{R}$.

The orem 1. If $f: \mathbf{R} \rightarrow \mathbf{C}$ is continuous and satisfies (1.1) then exactly one of the following holds:

(i) $f(x)=B$ for some complex constant $B$ and all $x$ in $\mathbf{R}$,

(ii) $f(x)=A x+B$ for some complex constants $A \neq 0, B$, and all $x$ in $\mathbf{R}$,

(iii) $f(x)=A e(\theta x)+B$ for some complex constants $A \neq 0, B$, a real constant $\theta \neq 0$ and all $x$ in $\mathbf{R}$. (We write $e(t)=e^{2 \pi i t}$.)

A useful class of mappings which satisfy (1.1) is the class of weakly 1-quasisymmetric embeddings of $\mathbf{R}$ into $\mathbf{C}$. The notion of a weakly quasisymmetric embedding was introduced by Tukia and Väisälä [TV]. In general if $\left(\mathscr{X}_{1}, d_{1}\right)$ and $\left(\mathscr{X}_{2}, d_{2}\right)$ are metric spaces, an embedding $f: \mathscr{X}_{1} \rightarrow \mathscr{X}_{2}$ is weakly $H$-quasisymmetric if $H \geqq 1$ is a constant such that

whenever

$$
d_{2}\{f(x), f(y)\} \leqq H d_{2}\{f(x), f(z)\}
$$

$$
d_{1}(x, y) \leqq d_{1}(x, z) .
$$

While an injective mapping $f: \mathbf{R} \rightarrow \mathbf{C}$ which satisfies (1.1) need not be weakly 1-quasisymmetric, it is clear that any weakly 1-quasisymmetric embedding must satisfy (1.1). Furthermore, if $f: \mathbf{R} \rightarrow \mathbf{C}$ is a weakly 1-quasisymmetric embedding then $f$ is unbounded [TV, (2.1) and (2.16)]. It will be convenient to combine these remarks with Theorem 1 .

* Research of the second author was supported by the National Science Foundation, DMS-8501941. 
Corollary 2. If $f: \mathbf{R} \rightarrow \mathbf{C}$ is a weakly 1-quasisymmetric embedding then $f(x)=$ $A x+B$ for some complex constants $A \neq 0, B$, and all $x$ in $\mathbf{R}$.

The statement of Corollary 2 is used in [M] to establish the existence of uniformly quasiconformal groups acting on $\mathbf{R}^{n}, n \geqq 3$, which have small dilatation. We remark that weakly 1-quasisymmetric embeddings of $\mathbf{R}$ into $\mathbf{R}^{n}, n \geqq 3$, need not be affine. This can be seen by considering an embedding

whose image is a helix.

$$
f(x)=(x, \cos x, \sin x)
$$

\section{Mapping $\mathbf{Z}$ into $\mathrm{C}$}

Throughout this section we assume that $f: \mathbf{Z} \rightarrow \mathbf{C}$ satisfies the identity

$$
|f(n+l)-f(n)|=|f(n-l)-f(n)|
$$

for all integers $l$ and $n$. This is equivalent to the requirement that for each fixed integer $l \geqq 1$ the function

$$
n \rightarrow|f(n+l)-f(n)|
$$

is periodic with period $l$. In particular,

$$
n \rightarrow|f(n+1)-f(n)|
$$

is constant. If $|f(1)-f(0)|=0$ then $f$ is constant on $\mathbf{Z}$, a case we no longer need to consider. Therefore we assume that $|f(1)-f(0)| \neq 0$ and then we may also assume that $|f(1)-f(0)|=1$.

For each integer $n$ we define

and

$$
\begin{aligned}
& \delta(n)=f(n+1)-f(n), \\
& \mu(n)=\delta(n+1) \overline{\delta(n),} \\
& \alpha(n)=\mathfrak{R}\{\mu(n)\},
\end{aligned}
$$

$$
\varepsilon(n)=\operatorname{sgn}(\mathfrak{I}\{\mu(n)\}) .
$$

From our previous assumptions we have

and

$$
|\delta(n)|=|\mu(n)|=1
$$

$$
\mu(n)=\alpha(n)+i \varepsilon(n)\left\{1-\alpha(n)^{2}\right\}^{1 / 2}
$$

for all $n$.

Lemma 3. The function $n \rightarrow \alpha(n)$ has period 2.

Proof. By hypothesis the function

$$
n \rightarrow|f(n+2)-f(n)|^{2}
$$


has period 2. We also have

$$
\begin{aligned}
|f(n+2)-f(n)|^{2} & =|\delta(n+1)+\delta(n)|^{2} \\
& =|\delta(n+1)|^{2}+2 \Re\{\delta(n+1) \overline{\delta(n)}\}+|\delta(n)|^{2} \\
& =2+2 \alpha(n),
\end{aligned}
$$

which proves the lemma.

Using Lemma 3 and the obvious fact that $-1 \leqq \alpha(n) \leqq 1$ for all $n$, we consider three separate cases.

Case 1: $\alpha(0)^{2}=\alpha(1)^{2}=1$,

Case 2: $\alpha(0)^{2}<1$ and $\alpha(1)^{2}<1$,

Case 3: $\alpha(0)^{2}<1$ and $\alpha(1)^{2}=1$ or $\alpha(0)^{2}=1$ and $\alpha(1)^{2}<1$.

In Case 1 we have $\mu(n)=\alpha(n)$ and therefore $\mu$ has period 2 . It remains to show that $\mu$ is also periodic in the other cases.

Lemma 4. In Case 2 the functions $n \rightarrow \varepsilon(n)$ and $n \rightarrow \mu(n)$ both have period 6 .

Proof. By hypothesis the function

$$
n \rightarrow|f(n+3)-f(n)|^{2}
$$

has period 3. Now, we have

$$
\begin{aligned}
|f(n+3)-f(n)|^{2} & =|\delta(n+2)+\delta(n+1)+\delta(n)|^{2} \\
& =|\delta(n+2) \overline{\delta(n+1)} \delta(n+1) \overline{\delta(n)}+\delta(n+1) \overline{\delta(n)}+1|^{2} \\
& =|1+\mu(n)+\mu(n) \mu(n+1)|^{2} \\
& =3+2 \Re\{\mu(n)+\mu(n+1)+\mu(n) \mu(n+1)\} \\
& =3+2\{\alpha(n)+\alpha(n+1)+\alpha(n) \alpha(n+1) \\
& \left.-\varepsilon(n) \varepsilon(n+1)\left(1-\alpha(n)^{2}\right)^{1 / 2}\left(1-\alpha(n+1)^{2}\right)^{1 / 2}\right\} .
\end{aligned}
$$

By Lemma 3 the function

$$
\alpha(n)+\alpha(n+1)+\alpha(n) \alpha(n+1)
$$

is constant and, in Case 2,

$$
\left(1-\alpha(n)^{2}\right)^{1 / 2}\left(1-\alpha(n+1)^{2}\right)^{1 / 2}
$$

is a nonzero constant. Hence we find that

$$
n \rightarrow \varepsilon(n) \varepsilon(n+1)
$$

has period 3. It follows that

$$
\{\varepsilon(n) \varepsilon(n+1)\}\{\varepsilon(n+1) \varepsilon(n+2)\}\{\varepsilon(n+2) \varepsilon(n+3)\}=\varepsilon(n) \varepsilon(n+3)
$$


is a constant function of $n$. Of course this constant is +1 or -1 , so

$$
\begin{aligned}
1 & =(\varepsilon(n) \varepsilon(n+3))^{2} \\
& =\varepsilon(n) \varepsilon(n+3) \varepsilon(n+3) \varepsilon(n+6) \\
& =\varepsilon(n) \varepsilon(n+6) .
\end{aligned}
$$

This shows that $n \rightarrow \varepsilon(n)$ has period 6. Finally, (2.3) and Lemma 3 imply that $n \rightarrow \mu(n)$ has period 6 .

Lemma 5. In Case 3 the functions $n \rightarrow \varepsilon(n)$ and $n \rightarrow \mu(n)$ both have period 8 .

Proof. We assume that $\alpha(0)^{2}<1$ and $\alpha(1)^{2}=1$. Then $\varepsilon(n)=0$ for odd integers $n$, so

$$
\varepsilon(2 m+1)=\varepsilon(2 m+1+8)
$$

is trivial. Thus we must show that

$$
\varepsilon(2 m)=\varepsilon(2 m+8)
$$

for all integers $m$.

By hypothesis the function

$$
m \rightarrow|f(2 m+4)-f(2 m)|^{2}
$$

has period 2. As in the proof of Lemma 4, we expand the right-hand side of (2.4) into terms involving $\alpha$ and $\mu$. We find that

Of course

$$
\begin{aligned}
|f(2 m+4)-f(2 m)|^{2} & =4+2\{\alpha(2 m)+\alpha(2 m+1)+\alpha(2 m+2)\} \\
& +2 \Re\{\mu(2 m) \mu(2 m+1)+\mu(2 m+1) \mu(2 m+2)\} \\
& +2 \mathfrak{R}\{\mu(2 m) \mu(2 m+1) \mu(2 m+2)\} .
\end{aligned}
$$

$$
m \rightarrow \alpha(2 m) \text { and } m \rightarrow \alpha(2 m+1)
$$

are constant. Since we are in Case 3,

and

$$
m \rightarrow \mathfrak{R}\{\mu(2 m) \mu(2 m+1)\}=\alpha(2 m) \alpha(2 m+1)
$$

$$
m \rightarrow \mathfrak{R}\{\mu(2 m+1) \mu(2 m+2)\}=\alpha(2 m+1) \alpha(2 m+2)
$$

are also constant. It follows that

$$
m \rightarrow \mathfrak{R}\{\mu(2 m) \mu(2 m+1) \mu(2 m+2)\}
$$

must have period 2. But in Case 3,

$$
\begin{gathered}
\mathfrak{R}\{\mu(2 m) \mu(2 m+1) \mu(2 m+2)\} \\
=\alpha(2 m) \alpha(2 m+1) \alpha(2 m+2)-\varepsilon(2 m) \varepsilon(2 m+2) \alpha(2 m+1)\left(1-\alpha(2 m)^{2}\right) .
\end{gathered}
$$

From our previous remarks and the fact that

$$
m \rightarrow\left(1-\alpha(2 m)^{2}\right)
$$


is a nonzero constant, we conclude that

$$
m \rightarrow \varepsilon(2 m) \varepsilon(2 m+2)
$$

has period 2. Thus for every integer $m$,

$$
\begin{aligned}
1 & =(\{\varepsilon(2 m) \varepsilon(2 m+2)\}\{\varepsilon(2 m+2) \varepsilon(2 m+4)\})^{2} \\
& =\{\varepsilon(2 m) \varepsilon(2 m+4)\}\{\varepsilon(2 m+4) \varepsilon(2 m+8)\} \\
& =\varepsilon(2 m) \varepsilon(2 m+8),
\end{aligned}
$$

and so $2 m \rightarrow \varepsilon(2 m)$ has period 8 . The corresponding result for $\mu$ follows from (2.3) and Lemma 3.

Next we suppose that $f: \mathbf{Z} \rightarrow \mathbf{C}$ satisfies (2.2), $|f(1)-f(0)|=1$, and that the corresponding function $\mu$ has period $p$, where $p \geqq 1$.

Theorem 6. Let $q$ be an integer. Then the function

$$
n \rightarrow f(q+p n)
$$

satisfies exactly one of the following conditions:

(i) $f(q+p n)=B$ for some complex constant $B$ and all $n \in \mathbf{Z}$,

(ii) $f(q+p n)=A n+B$ for complex constants $A \neq 0, B$, and all $n \in \mathbf{Z}$,

(iii) $f(q+p n)=A e(\theta n)+B$ for complex constants $A \neq 0, B$, a real constant $\theta$ with $0<\theta<1$, and all $n \in \mathbf{Z}$.

Proof. Since $\mu$ has period $p$ it follows that

$$
\gamma=\prod_{j=0}^{p-1} \mu(m+j)
$$

is a constant function of $m$ and of course $|\gamma|=1$. Therefore

and, replacing $m$ by $m-p$,

$$
\begin{aligned}
\delta(m+p) & =\left\{\prod_{j=0}^{p-1} \mu(m+j)\right\} \delta(m) \\
& =\gamma \delta(m)
\end{aligned}
$$

Thus we have

$$
\delta(m-p)=\gamma^{-1} \delta(m) .
$$

for all integers $m$ and $n$.

$$
\delta(m+p n)=\gamma^{n} \delta(m)
$$

If $n \geqq 1$ we have

$$
\begin{aligned}
f(q+p n)-f(q) & =\sum_{j=0}^{p n-1} \delta(q+j) \\
& =\sum_{k=0}^{n-1} \sum_{l=0}^{p-1} \delta(q+p k+l) \\
& =\sum_{k=0}^{n-1} \sum_{l=0}^{p-1} \gamma^{k} \delta(q+l) \\
& =\{f(q+p)-f(q)\} \sum_{k=0}^{n-1} \gamma^{k} .
\end{aligned}
$$


If $f(q+p)-f(q)=0$ then $n \rightarrow f(q+p n)$ clearly satisfies condition (i). If

then

$$
f(q+p)-f(q) \neq 0 \text { and } \gamma=1
$$

$$
f(q+p n)=f(q)+\{f(q+p)-f(q)\} n
$$

and an identical formula holds for $n \leqq 0$. Thus $f$ satisfies condition (ii). If

$$
f(q+p)-f(q) \neq 0 \text { and } \gamma \neq 1
$$

we may write $\gamma=e(\theta), 0<\theta<1$, and

$$
f(q+p n)=f(q)+\{f(q+p)-f(q)\}\left\{\frac{e(\theta n)-1}{e(\theta)-1}\right\} .
$$

This formula also extends easily to $n \leqq 0$. Of course (2.5) shows that $f$ satisfies condition (iii).

We note that the conclusion of Theorem 6 continues to hold as stated if we drop the assumption that $|f(1)-f(0)|=1$ and set $p=24$. Also, we have only used the fact that (2.2) has period $l$ for $l=1,2,3$ and 4 .

\section{Proof of Theorem 1}

Let $f: \mathbf{R} \rightarrow \mathbf{C}$ be continuous and satisfy (1.1). Clearly we may assume that $f$ is not constant on $\mathbf{R}$. For each $\alpha>0$ the function $n \rightarrow f\left((24)^{-1} \alpha n\right)$ maps $\mathbf{Z}$ into $\mathrm{C}$ and satisfies (2.1). By Theorem 6 and the remarks following that theorem, the function $n \rightarrow f(\alpha n)$ must be one of the three types described in Theorem 6. It will be convenient to formalize these observations as follows. For each $\alpha>0$ we define $f_{\alpha}: \mathbf{Z} \rightarrow \mathbf{C}$ by $f_{\alpha}(n)=f(\alpha n)$. Then for each $\alpha>0$ the function $f_{\alpha}$ satisfies exactly one of the conditions:

(i') $f_{\alpha}(n)=B(\alpha)$ for some complex number $B(\alpha)$ and all $n \in \mathbf{Z}$,

(ii') $f_{\alpha}(n)=A(\alpha) n+B(\alpha)$ for some complex numbers $A(\alpha) \neq 0, B(\alpha)$, and all $n \in \mathbf{Z}$,

(iii') $f_{\alpha}(n)=A(\alpha) e(\theta(\alpha) n)+B(\alpha)$ for some complex numbers $A(\alpha) \neq 0, B(\alpha)$, some real number $\theta(\alpha)$ with $0<\theta(\alpha)<1$, and all $n \in \mathbf{Z}$.

Since $f$ is not constant there are distinct real numbers $x_{1}$ and $x_{2}$ such that $f\left(x_{1}\right) \neq$ $f\left(x_{2}\right)$. Then by the continuity of $f$ there exists $\eta>0$ such that $f\left(y_{1}\right) \neq f\left(y_{2}\right)$ whenever $\left|x_{1}-y_{1}\right|<\eta$ and $\left|x_{2}-y_{2}\right|<\eta$. Next we fix a choice of $\alpha$ in the interval $0<\alpha<\frac{1}{2} \eta$. It follows that

and

$$
\left|x_{1}-\alpha\left[\frac{x_{1}}{\alpha}\right]\right|<\eta
$$

$$
\left|x_{2}-\alpha\left[\frac{x_{2}}{\alpha}\right]\right|<\eta,
$$


where $[\xi]$ is the integer part of the real number $\xi$. Therefore

$$
f_{\alpha}\left(\left[\frac{x_{1}}{\alpha}\right]\right) \neq f_{\alpha}\left(\left[\frac{x_{2}}{\alpha}\right]\right),
$$

which shows that for our choice of $\alpha$ the function $f_{\alpha}$ must have the form (ii') or (iii').

Let $\beta=2^{-m} \alpha$ where $m \geqq 1$ is an integer. Since $0<\beta<\frac{1}{2} \eta$ we see that $f_{\beta}$ must also have the form (ii') or (iii'). In fact, we claim that $f_{\alpha}$ and $f_{\beta}$ are either both of the form (ii') or both of the form (iii'). To see this we note that

$$
f_{\alpha}(n)=f_{\beta}\left(2^{m} n\right),
$$

for all $n \in \mathbf{Z}$. If $f_{\alpha}$ has the form (ii') then $f_{\alpha}$ is unbounded, hence $f_{\beta}$ is unbounded and therefore $f_{\beta}$ must also have the form (ii'). Conversely, if $f_{\beta}$ has the form (ii') then $f_{\beta}$ is unbounded on the subsequence $\left\{2^{m} n: n \in \mathbf{Z}\right\}$. Thus $f_{\alpha}$ is unbounded and has the form (ii').

Now suppose that $f_{\alpha}$ and $f_{\beta}$ both have the form (ii'). Then we may write (3.1) as

$$
A(\alpha) n+B(\alpha)=A\left(2^{-m} \alpha\right) 2^{m} n+B\left(2^{-m} \alpha\right)
$$

for all $n \in \mathbf{Z}$. Setting $n=0$ and $n=1$ it follows that

and

$$
B(\alpha)=B\left(2^{-m} \alpha\right)
$$

$$
A(\alpha)=A\left(2^{-m} \alpha\right) 2^{m} .
$$

Since $A(\alpha) \neq 0$ and $A\left(2^{-m} \alpha\right) \neq 0$ we find that

or

$$
f_{\beta}(n)=A(\alpha) 2^{-m} n+B(\alpha)
$$

$$
f\left(\alpha 2^{-m} n\right)=A(\alpha) 2^{-m} n+B(\alpha)
$$

for all $n \in \mathbf{Z}$. Let

$$
\mathscr{D}=\left\{2^{-m} n: m \in \mathbf{Z}, n \in \mathbf{Z} \text { and } m \geqq 1\right\},
$$

so that $\mathscr{D}$ is dense in $\mathbf{R}$. We have

$$
f(\alpha x)=A(\alpha) x+B(\alpha)
$$

for all $x$ in $\mathscr{D}$ by (3.2) and therefore (3.4) holds for all $x$ in R. This shows that $f$ has the form (ii) in the statement of Theorem 1.

Finally, we suppose that $f_{\alpha}$ and $f_{\beta}$ both have the form (iii'). Then

$$
A(\alpha) e(\theta(\alpha) n)+B(\alpha)=A\left(2^{-m} \alpha\right) e\left(\theta\left(2^{-m} \alpha\right) 2^{m} n\right)+B\left(2^{-m} \alpha\right)
$$

for all $n \in \mathbf{Z}$. Of course $0<\theta(\alpha)<1$ and therefore $\theta\left(2^{-m} \alpha\right) 2^{m}$ is not an integer. By computing the mean value

$$
\lim _{N \rightarrow \infty} N^{-1} \sum_{n=1}^{N} f_{\alpha}(n)
$$


we see that

$$
B(\alpha)=B\left(2^{-m} \alpha\right)
$$

Then we set $n=0$ in (3.5) and find that

$$
A(\alpha)=A\left(2^{-m} \alpha\right)
$$

Let $g: \mathbf{R} \rightarrow \mathbf{C}$ be the continuous function

$$
g(x)=A(\alpha)^{-1}\{f(\alpha x)-B(\alpha)\},
$$

and for each integer $m \geqq 1$ let

$$
\mathscr{D}_{m}=\left\{2^{-m} n: n \in \mathbf{Z}\right\} .
$$

Then $\mathscr{D}_{1} \subseteq \mathscr{D}_{2} \subseteq \ldots$, each $\left(\mathscr{D}_{m},+\right)$ is a subgroup of $(\mathbf{R},+), \mathscr{D}=\bigcup_{m=1}^{\infty} \mathscr{D}_{m}$, and $(\mathscr{D},+)$ is a dense subgroup. Since

$$
\begin{aligned}
g\left(2^{-m} n\right) & =A(\alpha)^{-1}\left\{f_{\beta}(n)-B(\alpha)\right\} \\
& =e\left(\theta\left(2^{-m} \alpha\right) n\right),
\end{aligned}
$$

we conclude that $g$ restricted to $\mathscr{D}$ is a homomorphism into the circle group $\mathbf{T}=\{\zeta \in \mathbf{C}:|\zeta|=1\}$. Since $g$ is continuous on $\mathbf{R}$ it follows that $g: \mathbf{R} \rightarrow \mathbf{T}$ is a homomorphism, that is, $g$ is a group character. Hence

$$
g(x)=e(\theta \alpha x)
$$

for some real $\theta \neq 0$ and all real $x$. Now (3.6) shows that $f$ has the form (iii) in the statement of the Theorem. (That $g$ must have the shape (3.7) is proved, for example, in $[R, p .12]$.)

\section{References}

[M] McKemie, M. J.: Quasiconformal groups with small dilatation. - Ann. Acad. Sci. Fenn. Ser. A I Math. 12, 1987, 95-118.

[R] Rudin, W.: Fourier analysis on groups. - Interscience Publishers, John Wiley \& Sons, New York-London-Sydney, 1967.

[TV] TUkIA, P., and J. VÄIsÄLÄ: Quasisymmetric embeddings of metric spaces. - Ann. Acad. Sci. Fenn. Ser. A I Math. 5, 1980, 97-114.

University of Missouri-Rolla

Department of Mathematics and Statistics

Rolla, Missouri 65401-0249

U.S.A.
University of Texas

Department of Mathematics

Austin, Texas 78712

U.S.A.

Received 6 August 1986 